\title{
Acoustic vortices in inhomogeneous media
}

\author{
Xu-Dong Fan, Zheguang Zou, and Likun Zhang @* \\ National Center for Physical Acoustics and Department of Physics and Astronomy, \\ University of Mississippi, University, Mississippi 38677, USA
}

(Received 11 May 2019; published 4 November 2019)

\begin{abstract}
We observe a series of unstable and dynamic behaviors of acoustic vortices propagating in stratified inhomogeneous media. These behaviors include bending, stretching, distorting, focusing, and untwisting of the vortices, migration of singular points, and reversal of energy flux and angular momentum. We gain insight into how these behaviors result from the stratification. The results will be useful for applications of acoustic vortices in communication and particle manipulations in inhomogeneous media such as oceans and biomedical tissues. Our work opens up the study of the propagation of acoustic vortices in complex media.
\end{abstract}

DOI: 10.1103/PhysRevResearch.1.032014

Singularities and wave vortices exist in various natural phenomena and applications $[1,2]$. With a spiral phase $\exp (\operatorname{im} \phi)$ proportional to the azimuth angle $\phi$, the vortices carry orbital angular momentum (OAM), as investigated in both acoustical (linear [3-6] and nonlinear [7-9]) and quantum (optical $[10,11]$ and electron [12-14]) fields. The integer $m$ is the topological charge of the vortex and the field has a null at the core. It is of fundamental and practical interests to study the propagation of wave vortices and transport of OAM in heterogeneous media. The propagation of optical vortices through an inhomogeneous medium was considered in the scalar approximation in Ref. [15], where a theoretical approach was proposed to address the problem of singular fields in the context of improving optical systems in the turbulent atmosphere.

To our knowledge, prior studies on acoustic vortices were limited to homogeneous media, with an exception for simulating the paraxial propagation of nonlinear vortices in weakly heterogeneous media [9]. Acoustic vortices generated by phased spiral sources or physically spiral sources [16] can be used in many applications, especially in particle manipulations (e.g., Refs. [4-6,17-30]), underwater navigation [31], and communications [32,33]. When considering practical situations or applications, the media commonly have spatially varying parameters in various areas. For example, in deep ocean environments, the sound speed is a function of depth, resulting from temperature and salinity stratification, and pressure variation [34,35]. In biomedical ultrasound, the sound speed has a large variation crossing tissues (see, e.g., Refs. [36-38]). There are interests to use vortex beams for ultrasonic alignment [3], imaging [39], therapy [40], and underwater navigation [31]. The propagation of acoustic vortices

\footnotetext{
*zhang@olemiss.edu
}

Published by the American Physical Society under the terms of the Creative Commons Attribution 4.0 International license. Further distribution of this work must maintain attribution to the author(s) and the published article's title, journal citation, and DOI. in inhomogeneous media is hence fundamental and vital to address, yet remains to be explored.

In this Rapid Communication, we open up the study by investigating acoustic singularities and vortices in stratified inhomogeneous media. We conduct numerical simulations and theoretical analysis addressing how the vortex field and linear/angular momentum evolves in stratified media. We examine the cases of both linear and nonlinear stratification in ultrasound fields and in ocean environments.

Here, the shear components have not been included. For propagation in a viscoelastic medium and applications in ultrasound imaging, the shear component can be considered separately if needed since the velocities of shear waves are normally two orders smaller than the compressional waves in most biomedical materials [41]. The shear waves may be circularly polarized to carry spin angular momentum (SAM). In optics, for an elliptically polarized vortex beam transmitting in an inhomogeneous medium [42-46] or through a sharp interface between two media $[43,44]$, the coupling between SAM and OAM results in spin-Hall effects; there can also be orbital-Hall effects for optical vortices $[47,48]$.

Propagation in linearly stratified fluids. We start by simulating the propagation of ultrasonic vortex fields in a linearly stratified fluid, where the sound speed is $c=c_{0}-G z$, such as that considered in Refs. [35,38]. The gradient is as large as $G=58 \mathrm{~m} / \mathrm{s}$ per $\mathrm{mm}$, which enhances the stratification effect in a short propagating distance (that saves the simulation load). The gradient is relevant to the sharp change through boundaries between two biomedical materials such as tumors [41]. A weaker gradient would play a role in a longer propagation distance as we will illustrate later in another example. We use the typical sound speed in fluids $c_{0}=1500 \mathrm{~m} / \mathrm{s}$ at the vortex source center and neglect the variation of fluid density. A finite plane source with a diameter of $15 \lambda$ is used, which is at the order of 1-in.-diam transducer if for $1 \mathrm{MHz}$ ultrasound is to have a wavelength $\lambda=1.5 \mathrm{~mm}$.

The simulated source profile has a topological charge of -1 in polar coordinates $(\rho, \phi), \psi=J_{1}(\mu \rho) e^{-i \phi} e^{-i \omega t}$, where $J_{1}$ is the first-order Bessel function, $\omega$ is the angular frequency, and $\mu$ is the transverse wave number (chosen to be half of the 

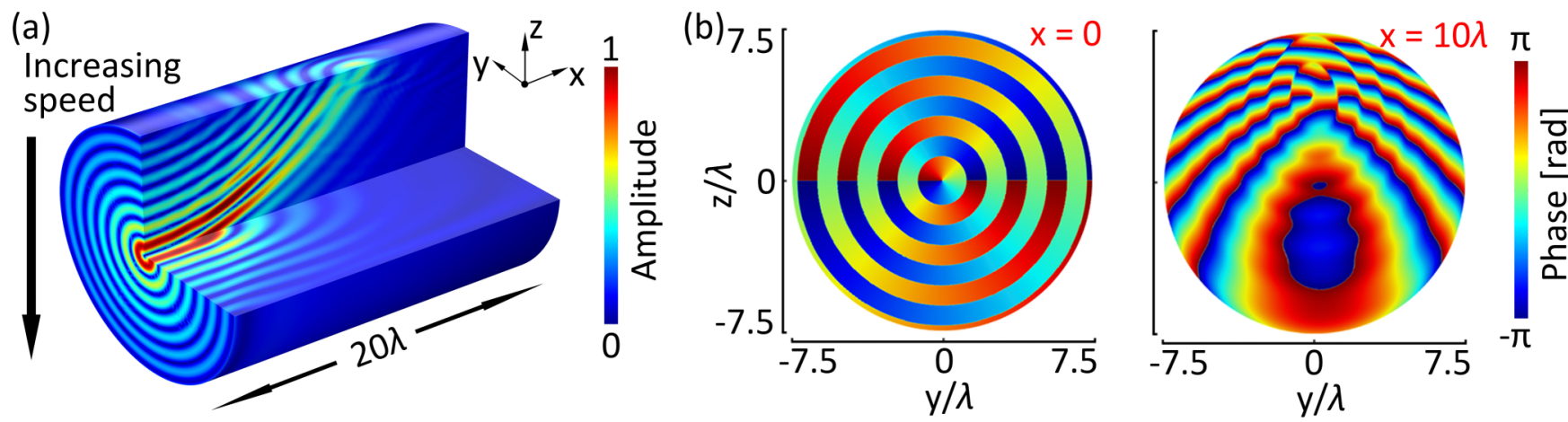

FIG. 1. (a) Simulation of vortex waves bending in a stratified medium. (b) Stretching and distorting of the phase on $y$ - $z$ cross sections at different propagating distances $x$.

total wave number $\left.k=\omega / c_{0}\right)$. The simulation is conducted with a finite-element method based on COMSOL MULTIPHYSICS software. Radiation boundary conditions are applied to the outer boundaries of the calculation domain (a cylinder with $15 \lambda$ in diameter and $20 \lambda$ in length) to model the propagation in a free space without reflection. The source is located at the $x=0$ plane [Fig. 1(a)].

Figure 1(a) shows the simulated three-dimensional wave amplitude. The vortex beam bends upwards towards the $+z$ direction, as expected due to the refraction. Figure 1(b) displays the phase distortions on the $y-z$ plane at different $x$. Overall, the vortices bend upwards and are stretched in the stratified direction [49]. We then examine the evolution of the wave amplitude during the propagation [Fig. 2(a)]. Unexpectedly, we find the amplitude is asymmetric in the nonstratified $y$ direction (relative to $y=0$ ) [49], arising from the fact that the vortices rotate clockwise to have their propagation direction along or against the upward refraction, depending on the positive or negative $y$ values, respectively [Fig. 2(b)].

We characterize the trajectory of the vortex center [Fig. 2(c)], which is identified from the singular point of the phase distribution. The singular trajectory is compared with a horizontally emitted eigenray from a point source (black dashed line), which is an arc of a circle $c_{0} / G$ in a linearly stratified medium [35]. The results show that the singular trajectory does not lie on the eigenray of the point source. For comparison, we further simulate a beam from the same source but without the vortex phases [i.e., a zero-order
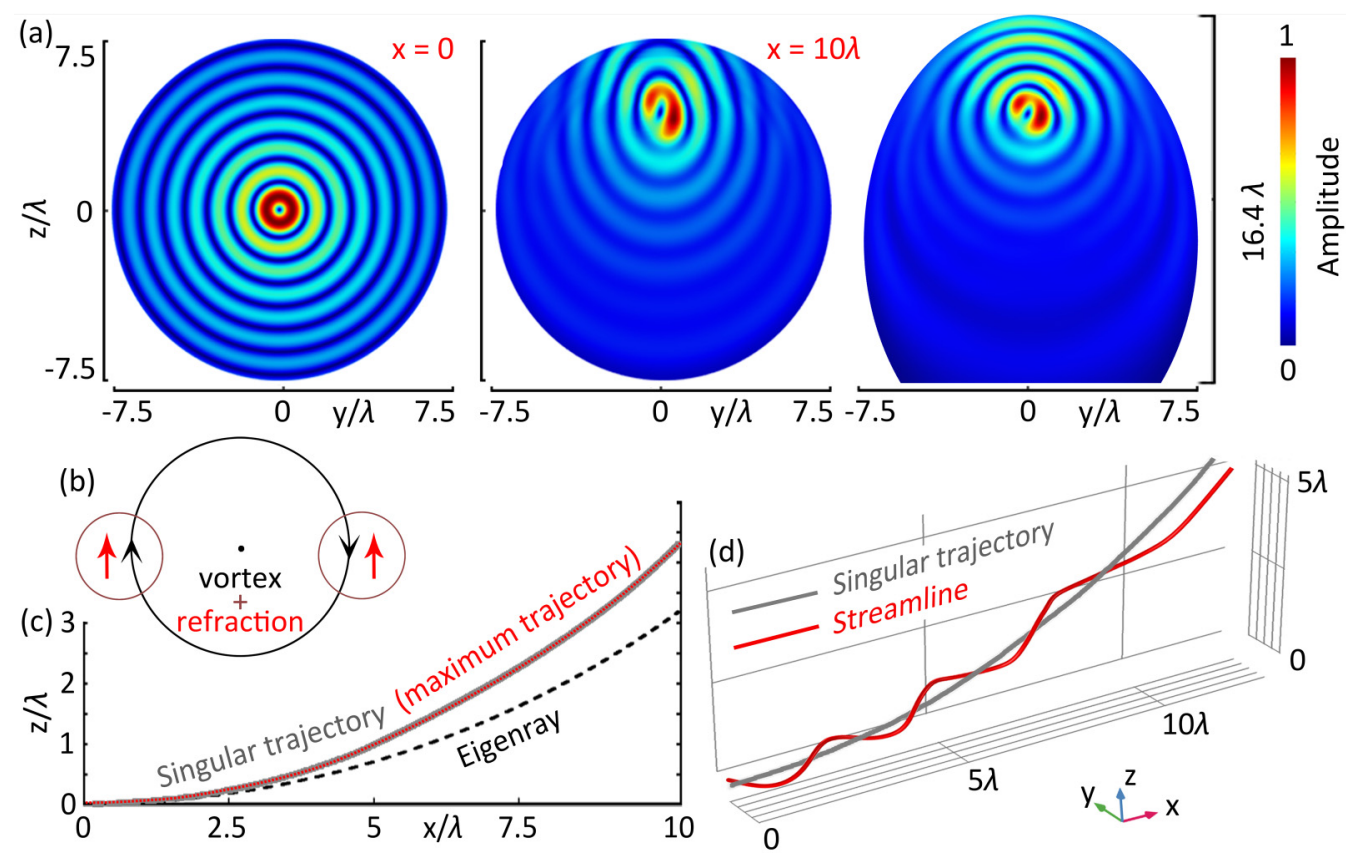

FIG. 2. (a) Stretching and distorting of amplitude on $y-z$ cross sections at $x=0$ (left) and $10 \lambda$ (middle), and on the cross section normal to the singular trajectory through the singular point at $x=10 \lambda$. (b) Illustration of the mechanism of amplitude asymmetry. Red arrows represent the refraction direction by stratification. (c) Singular trajectory on the $z-x$ plane (gray solid line) and its comparison with a horizontally emitted eigenray (black dashed line) and a maximum amplitude trajectory from a zero-order Bessel beam propagating in the same media (red dotted line). (d) Three-dimensional energy flux and vortex center. A three-dimensional streamline starting from $(0,0,0.25 \lambda)$ and twisting around the vortex center (singular trajectory) is untwisted from certain distances. 

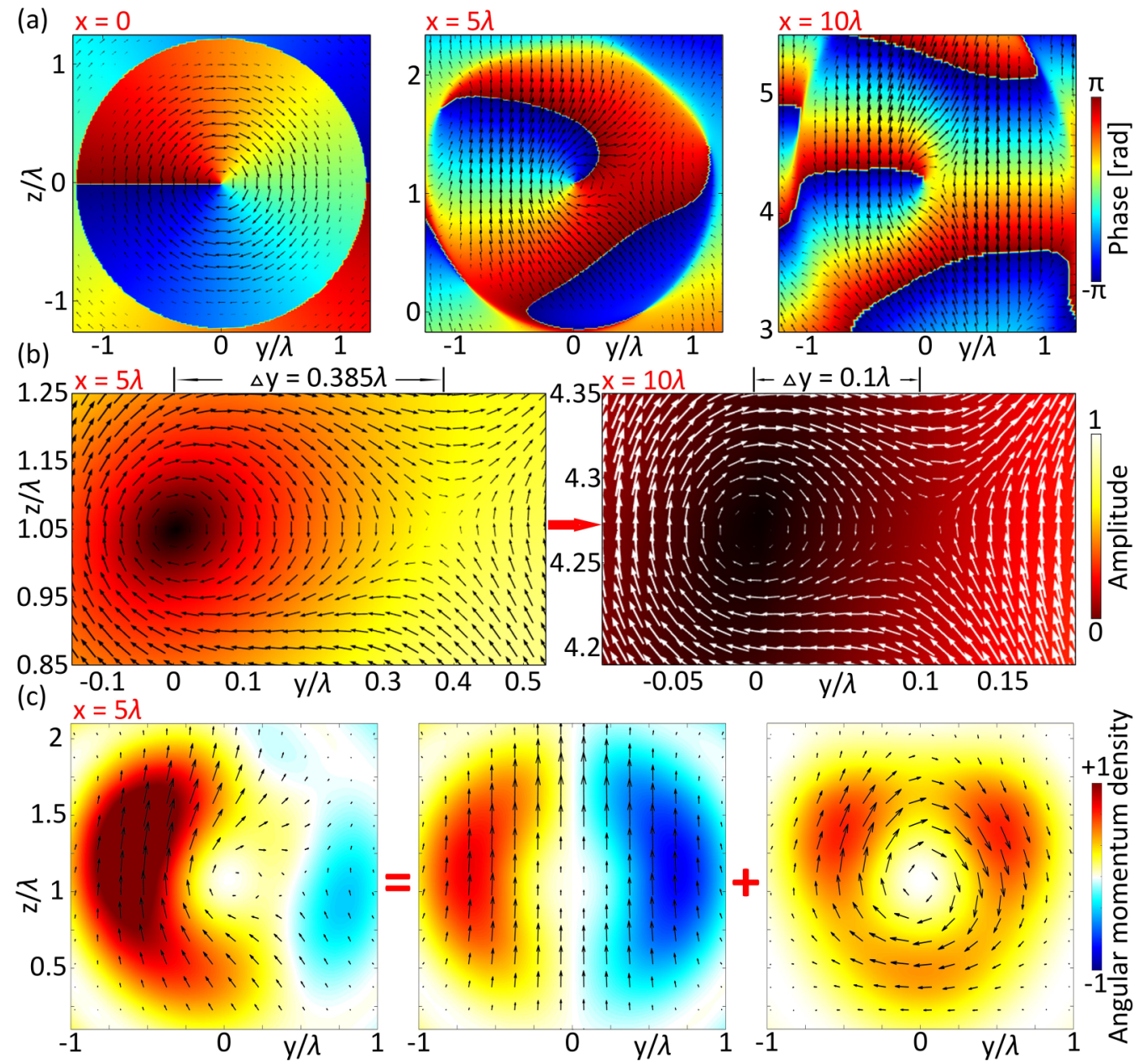

FIG. 3. Features of transverse energy flux (arrows): (a) Reversal from clockwise to counterclockwise, (b) migration of an additional singular point of saddle type towards the vortex center [zoom-in areas of panels in (a)], and (c) separation into two portions-the stratified effect where the flux is upwards (middle panel) and a reconstructed vortex (right-hand-side panel). Color plots in (a) show the phase distribution, in (b) show the wave amplitude, and in (c) show angular momentum density.

beam, $\left.\psi=J_{0}(\mu \rho) e^{-i \omega t}\right]$, where the trajectory of the central pressure maximum [Fig. 2(c)] does coincide with the singular trajectory of the vortices, revealing that the singular trajectory follows the path of a beam emitted by a finite-size source.

We now reveal the transport of energy by examining the time-averaged energy flux over a wave period, $\mathbf{S}=c^{2} \mathbf{g}$ [4], where the time-averaged momentum density $\mathbf{g}=\operatorname{Im}\left(\psi^{*} \nabla \psi\right)$ is calculated from the complex scalar field $\psi$ and its gradient that gives the velocity (Im represents the imaginary part and a prefactor is suppressed in the normalization of $\psi$ ). The flux is illustrated by one typical streamline in Fig. 2(d), showing that the energy flux is transformed from twisted into untwisted and simply bends upwards at a large distance. The transition results from the fact that, as the wave propagates and the vortex spreads, the upward energy flux due to the stratification gradually dominates.

The transverse flux at different propagating distances $x$ is further shown in Fig. 3(a) by arrow plots. We find that the flux is reversed on the positive $y$ side as the wave propagates. The direction of energy flux corresponds to the gradient of phase of wave propagation [Fig. 3(a)]. Reversal of the flux implies the opposite propagation of the vortices, resulting from an additional upward energy flux by the refraction. The transition of the flux direction is at locations where the upward flux by refraction cancels with the clockwise vortex flux, leading to the emergence of another singular point where the transverse energy flux is zero [Fig. 3(b)]. This singular point of transverse energy flux is of saddle type, in contrast to the center point of the vortices. As the vortex beam propagates and spreads, the saddle point migrates towards the center point of the vortices [49]. Note that at this additional singular point, the pressure [Fig. 3(b)] and axial velocity are not zero, so there is still a flux along the propagating $x$ direction at these locations.

Even though the vortices are unstable and untwisted by the stratification as we have observed, we propose a way to individually visualize the vortex flow and the stratified effect by separating the transverse energy flux into two portions. The first portion is the background flux (denoted by $\mathbf{g}^{\mathrm{B}}$ ) obtained by taking the antisymmetrical (symmetrical) part 

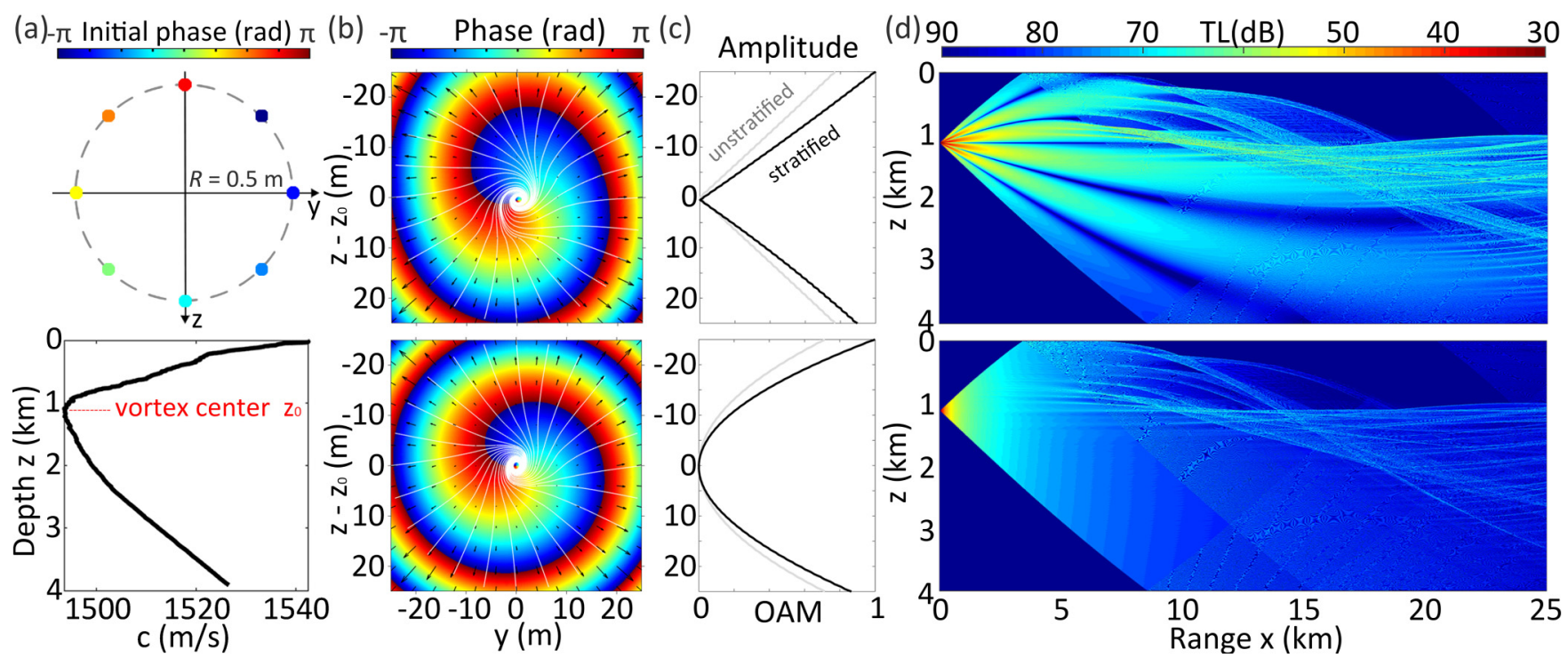

FIG. 4. Modeling the propagation of acoustic vortices in stratified oceans. (a) Vortex source array of identical amplitude $p_{0}$ (top panel), centered on the depth of the sound speed minimum $z_{0}=1115 \mathrm{~m}$ of the sound speed profile (bottom panel). (b) Vortex phases (color plots) and energy flux (black arrows and white streamlines) in the stratified ocean (top) and a comparison with propagation in an unstratified ocean (bottom). The resolution of the plot is $0.01 \mathrm{~m}$ (1/10 of the wavelength). (c) Normalized pressure amplitude (top) and $x$ component of OAM density (bottom) as a function of depth at $x=3000 \mathrm{~m}$ and $y=0 \mathrm{~m}$. (d) Transmission loss (TL, see text) in the vertical $x-z$ plane $(y=0)$ simulated for the vortex array (top panel) and compared with a point source (bottom panel).

of the energy flux in the horizontal $y$ (vertical z) direction, namely,

$$
\begin{gathered}
\mathbf{g}_{y}^{\mathrm{B}}(y, z)=\left[\mathbf{g}_{y}(y, z)-\mathbf{g}_{y}(-y, z)\right] / 2, \\
\mathbf{g}_{z}^{\mathrm{B}}(y, z)=\left[\mathbf{g}_{z}(y, z)+\mathbf{g}_{z}(-y, z)\right] / 2 .
\end{gathered}
$$

The second portion is the vortex flux (denoted by $\mathbf{g}^{\mathrm{v}}$ ) obtained by taking the symmetrical (antisymmetrical) part of the energy flux in the vertical $y$ (horizontal $z$ ) direction, namely,

$$
\begin{aligned}
& \mathbf{g}_{y}^{\mathrm{V}}(y, z)=\left[\mathbf{g}_{y}(y, z)+\mathbf{g}_{y}(-y, z)\right] / 2, \\
& \mathbf{g}_{z}^{\mathrm{V}}(y, z)=\left[\mathbf{g}_{z}(y, z)-\mathbf{g}_{z}(-y, z)\right] / 2 .
\end{aligned}
$$

The separation is illustrated in Fig. 3(c), where the transverse energy flux in the left-hand-side panel is separated into a $u p$ ward background flux in the middle panel and a reconstructed clockwise vortex in the right-hand-side panel.

Lastly, we display the angular momentum density $\mathbf{j}=\mathbf{r} \times \mathbf{g}$ in Fig. 3(c) by its axial $x$ component (color plots), calculated from the momentum density $\mathbf{g}$ and the relative distance to the vortex center at the corresponding cross section. The total angular momentum density in the left-hand-side panel is a sum of angular momentum density in the middle panel and the reconstructed vortex in the right-hand-side panel. There is no contribution to the total angular momentum contained in the whole cross section from the refraction in the middle panel, where the angular momentum density is antisymmetric with respect to $y=0$. The total angular momentum contained in the original vortex is equal to the total angular momentum of the reconstructed vortex. The separation of the stratified part does not change the total angular momentum other than it redistributes the angular momentum density in the cross section.
Propagation in stratified oceans. We now simulate the propagation of acoustic vortices in the deep ocean environment for acoustic communication. The simulations are for a sound speed profile [Fig. 4(a)] calculated from temperature, salinity, and pressure measured in the World Ocean Circulation Experiment [50] in the North Atlantic Ocean $\left(24.5013^{\circ} \mathrm{N}\right.$, $47.5800^{\circ} \mathrm{W}$ ) on 3 August 1992 . The profile ranges from 1495 to $1525 \mathrm{~m} / \mathrm{s}$ (gradient less than $0.1 \mathrm{~m} / \mathrm{s}$ per m) with the minimum sound speed at a depth of $1115 \mathrm{~m}$, where the sound channel axis is formed by acoustic refraction [34]. The vortex beam is simulated by eight point sources located on a circle [Fig. 4(a)]. The eight sources have the same amplitude $p_{0}$ and $\pi / 4$ phase increment to form a vortex beam of topological charge 1 (i.e., $2 \pi$ phase change over a circle). The acoustic frequency is chosen at $15 \mathrm{kHz}$, which is the typical center frequency for midrange (10-25 km) acoustic communication [51]. Given that three-dimensional (3D) sound modeling in the context of oceans has a huge computational cost when using COMSOL (see, for example, Ref. [52]), we use the twodimensional (2D) BELLHOP model, which is a widely used ray-tracing ocean acoustic model [53]. We simulate the sound fields emitted by the eight individual sound sources [54]. The 3D sound field emitted by the vortex source follows from a sum of the fields simulated for the eight individual sound sources but the initial phase differences at the sources are accounted for in the sum. The superposed vortex fields should follow the feature of fields emitted by a finite-sized source.

The simulated three-dimensional propagation is shown in Fig. 4(b) by the acoustic phase and energy flux at a cross section of range $x=3000 \mathrm{~m}$. The results reveal that the ocean stratification deforms (vertically stretches) the vortices and introduces asymmetric features as we previously observed, though due to the relatively weak stratification the asymmetry is not as strong as the previous case. 
We further observe that both the pressure and OAM are enhanced when comparing with the case of no stratification [Fig. 4(c)]. The enhancement results from the opposite gradients of the stratification above and below the vortex center, which bend the sound beam and flux towards the depth of sound speed minimum (i.e., sound channel axis). This is a focusing effect, identical to the case for sources without a vortex feature [34]. The focusing effect does not exist in the linear gradient where the refraction simply bends the beam towards one direction.

The acoustic field in the vertical $x-z$ plane is further shown in the top panel of Fig. 4(d) for a long-range propagation $(25 \mathrm{~km})$ [55], where the transmission loss is TL = $-20 \log _{10}\left(p / p_{0}\right)$, with $p$ being the sound pressure amplitude. The results show that, while the sound rays are refracted towards the sound channel axis at the depth of the sound speed minimum, the vortex phase feature leads to a light and dark interference pattern in the field. We find that acoustic wave fields along the sound channel axis are singular only at a certain range $x$ before the presence of the acoustic refraction towards the channel axis or reflections from the surface and bottom. A comparison of the field with that emitted from a point source [bottom panel in Fig. 4(d)] reveals that, even though the ocean gradient is relatively weak, there is still a visible focus and deformation of the vortices over a long-range propagation, suggesting the significant impact of ocean stratification on the long-range propagation of acoustic vortices in stratified oceans.

Discussion. We have reported behaviors of acoustic singularities and vortices in stratified ultrasonic and underwater media. We found that the stratification feature leads to the distortion and complex behaviors of the vortices via the emergence of bending, distorting, focusing, and stretching of the fields, or even the reversal of the energy and momentum transports, and angular momentum. The vortex shapes and behaviors are different between the two cases we have examined. The nonlinear gradient leads to the vortex focusing towards the sound speed minimum, while the linear gradient simply bends the vortex to one direction. The reversal of energy flux and angular momentum appears only when the propagating distance or gradient is significant enough.

All of the observations herein suggest the complexity of the applications of vortices in inhomogeneous media in the contexts of oceans and ultrasound. For the purpose of underwater navigation and communication, it becomes challenging to decompose the vortices of different topological charges in the stratified ocean. The findings of distortion of the energy flux and angular momentum of vortices by the refraction can lead to different phenomena of vortex-based particle manipulations in inhomogeneous fluids, where the spatial gradient plays a central role [56]. One scenario is the particle manipulation and transport in the presence of a thermal gradient [57-59], where the distortion of the vortex propagation by the gradient as we have observed may need to be taken into account. It would also be interesting to examine in the stratified media for the orbital and spinning motion of particles due to the vortices by the phase gradient $[4-6,60]$ or by the local rotation of the velocity field $[61,62]$.

In the future, the current study can be extended to consider nonlinear effects, attenuation, a viscoelastic medium, and a three-dimensional heterogeneous medium. The behaviors reported herein are expected to be observed in other vortex fields. It would be useful to implement the existing numerical tools $[9,37]$ to describe the propagation of acoustic vortices in heterogeneous media. Experimental work to validate the behaviors would be valuable. One may use the setup of the ultrasound tank experiment in a stratified fluid [35], where the gradient is two orders smaller than the first example but several orders larger than oceans.

Another relevant problem is to investigate propagation in homogeneous anisotropic media, such as optical vortices transmitting in a uniaxial crystal, where the change in angular momentum depends on the orientation of the crystals [63-73], and the conservation of the total angular momentum (a sum of OAM and SAM) is broken by the anisotropy even for the beam transmitting along a crystal optical axis [74]. The discussions about homogeneous anisotropic media are beyond the scope of this Rapid Communication. The converse phenomenon of an anisotropic Bessel beam propagating in isotropic media would also be interesting $[75,76]$.

Acknowledgments. We acknowledge the funding support from the University of Mississippi via a start-up fund, and the funding support from the Gulf of Mexico program administrated by the National Academics of Sciences. We acknowledge discussions with Dr. Yun Jing and Dr. Juanjuan Gu at North Carolina State University.
[1] J. F. Nye and M. V. Berry, Dislocations in wave trains, Proc. R. Soc. London, Ser. A 336, 165 (1974).

[2] M. Berry, Making waves in physics, Nature (London) 403, 21 (2000).

[3] B. T. Hefner and P. L. Marston, An acoustical helicoidal wave transducer with applications for the alignment of ultrasonic and underwater systems, J. Acoust. Soc. Am. 106, 3313 (1999).

[4] L. Zhang and P. L. Marston, Angular momentum flux of nonparaxial acoustic vortex beams and torques on axisymmetric objects, Phys. Rev. E 84, 065601(R) (2011).

[5] L. K. Zhang and P. L. Marston, Acoustic radiation torque on small objects in viscous fluids and connection with viscous dissipation, J. Acoust. Soc. Am. 136, 2917 (2014).
[6] L. K. Zhang, Reversals of Orbital Angular Momentum Transfer and Radiation Torque, Phys. Rev. Applied 10, 034039 (2018).

[7] J.-L. Thomas and R. Marchiano, Pseudo Angular Momentum and Topological Charge Conservation for Nonlinear Acoustical Vortices, Phys. Rev. Lett. 91, 244302 (2003).

[8] R. Marchiano and J. L. Thomas, Synthesis and analysis of linear and nonlinear acoustical vortices, Phys. Rev. E 71, 066616 (2005).

[9] R. Marchiano, F. Coulouvrat, L. Ganjehi, and J. L. Thomas, Numerical investigation of the properties of nonlinear acoustical vortices through weakly heterogeneous media, Phys. Rev. E 77, 016605 (2008). 
[10] L. Allen, M. W. Beijersbergen, R. J. C. Spreeuw, and J. P. Woerdman, Orbital angular momentum of light and the transformation of Laguerre-Gaussian laser modes, Phys. Rev. A 45, 8185 (1992).

[11] M. R. Dennis, K. O'Holleran, and M. J. Padgett, Singular optics: Optical vortices and polarization singularities, Prog. Opt. 53, 293 (2009).

[12] M. Uchida and A. Tonomura, Generation of electron beams carrying orbital angular momentum, Nature (London) 464, 737 (2010).

[13] J. Verbeeck, H. Tian, and P. Schattschneider, Production and application of electron vortex beams, Nature (London) 467, 301 (2010).

[14] B. J. McMorran, A. Agrawal, I. M. Anderson, and A. A. Herzing, Electron vortex beams with high quanta of orbital angular momentum, Science 331, 192 (2011).

[15] V. P. Aksenov, V. V. Kolosov, V. A. Tartakovskii, and B. V. Fortes, Optical vortices in inhomogeneous media, Atmos. Oceanic Opt. 12, 912 (1999).

[16] X. Jiang, Y. Li, B. Liang, J.-C. Cheng, and L. Zhang, Convert Acoustic Resonances to Orbital Angular Momentum, Phys. Rev. Lett. 117, 034301 (2016).

[17] K. D. Skeldon, C. Wilson, M. Edgar, and M. J. Padgett, An acoustic spanner and its associated rotational Doppler shift, New J. Phys. 10, 013018 (2008)

[18] K. Volke-Sepúlveda, A. O. Santillán, and R. R. Boullosa, Transfer of Angular Momentum to Matter from Acoustical Vortices in Free Space, Phys. Rev. Lett. 100, 024302 (2008).

[19] C. E. M. Demore, Z. Yang, A. Volovick, S. Cochran, M. P. MacDonald, and G. C. Spalding, Mechanical Evidence of the Orbital Angular Momentum to Energy Ratio of Vortex Beams, Phys. Rev. Lett. 108, 194301 (2012).

[20] A. Anhäuser, R. Wunenburger, and E. Brasselet, Acoustic Rotational Manipulation Using Orbital Angular Momentum Transfer, Phys. Rev. Lett. 109, 034301 (2012).

[21] Z. Y. Hong, J. Zhang, and B. W. Drinkwater, Observation of Orbital Angular Momentum Transfer from Bessel-Shaped Acoustic Vortices to Diphasic Liquid-Microparticle Mixtures, Phys. Rev. Lett. 114, 214301 (2015).

[22] T. Wang, M. Ke, W. Li, Q. Yang, C. Qiu, and Z. Liu, Particle manipulation with acoustic vortex beam induced by a brass plate with spiral shape structure, Appl. Phys. Lett. 109, 123506 (2016).

[23] A. Marzo, M. Caleap, and B. W. Drinkwater, Acoustic Virtual Vortices with Tunable Orbital Angular Momentum for Trapping of Mie Particles, Phys. Rev. Lett. 120, 044301 (2018).

[24] D. Baresch, J. L. Thomas, and R. Marchiano, Orbital Angular Momentum Transfer to Stably Trapped Elastic Particles in Acoustical Vortex Beams, Phys. Rev. Lett. 121, 074301 (2018).

[25] X.-D. Fan and L. Zhang, Trapping Force of Acoustical Bessel Beams on a Sphere and Stable Tractor Beams, Phys. Rev. Applied 11, 014055 (2019).

[26] S. T. Kang and C. K. Yeh, Potential-well model in acoustic tweezers, IEEE Trans. Ultrason. Ferroelectr. Freq. Control 57, 1451 (2010).

[27] A. Marzo, S. A. Seah, B. W. Drinkwater, D. R. Sahoo, B. Long, and S. Subramanian, Holographic acoustic elements for manipulation of levitated objects, Nat. Commun. 6, 8661 (2015).

[28] D. Baresch, J.-L. Thomas, and R. Marchiano, Observation of a Single-Beam Gradient Force Acoustical Trap for Elastic
Particles: Acoustical Tweezers, Phys. Rev. Lett. 116, 024301 (2016).

[29] K. Melde, A. G. Mark, T. Qiu, and P. Fischer, Holograms for acoustics, Nature (London) 537, 518 (2016).

[30] M. Baudoin, J. C. Gerbedoen, A. Riaud, O. B. Matar, N. Smagin, and J. L. Thomas, Folding a focalized acoustical vortex on a flat holographic transducer: Miniaturized selective acoustical tweezers, Sci. Adv. 5, eaav1967 (2019).

[31] B. T. Hefner and B. R. Dzikowicz, A spiral wave front beacon for underwater navigation: Basic concept and modeling, J. Acoust. Soc. Am. 129, 3630 (2011).

[32] C. Z. Shi, M. Dubois, Y. Wang, and X. Zhang, Highspeed acoustic communication by multiplexing orbital angular momentum, Proc. Natl. Acad. Sci. USA 114, 7250 (2017).

[33] X. Jiang, B. Liang, J. C. Cheng, and C. W. Qiu, Twisted acoustics: Metasurface-enabled multiplexing and demultiplexing, Adv. Mater. 30, 1800257 (2018).

[34] W. H. Munk, Sound channel in an exponentially stratified ocean, with application to sofar, J. Acoust. Soc. Am. 55, 220 (1974).

[35] L. K. Zhang and H. L. Swinney, Sound propagation in a continuously stratified laboratory ocean model, J. Acoust. Soc. Am. 141, 3186 (2017)

[36] M. S. Soldevilla, M. F. McKenna, S. M. Wiggins, R. E. Shadwick, T. W. Cranford, and J. A. Hildebrand, Cuvier's beaked whale (Ziphius Cavirostris) head tissues: Physical properties and CT imaging, J. Exp. Biol. 208, 2319 (2005).

[37] J. Gu and Y. Jing, Numerical modeling of ultrasound propagation in weakly heterogeneous media using a mixed domain method, IEEE Trans. Biomed. Eng. 65, 1258 (2018).

[38] S. Schoen, Jr. and C. D. Arvanitis, Fast acoustic aberration correction with the angular spectrum approach, arXiv:1906.08156.

[39] T. Brunet, J. L. Thomas, and R. Marchiano, Transverse Shift of Helical Beams and Subdiffraction Imaging, Phys. Rev. Lett. 105, 034301 (2010).

[40] A. D. Maxwell, M. Bailey, B. W. Cunitz, M. Terzi, A Nikolaeva, S. Tsysar, and O. A. Sapozhnikov, Vortex beams and radiation torque for kidney stone management, J. Acoust. Soc. Am. 139, 2040 (2016).

[41] A. P. Sarvazyan, M. W. Urban, and J. F. Greenleaf, Acoustic waves in medical imaging and diagnostics, Ultrasound Med. Biol. 39, 1133 (2013).

[42] V. S. Liberman and B. Y. Zeldovich, Spin-orbit interaction of a photon in an inhomogeneous medium, Phys. Rev. A 46, 5199 (1992).

[43] K. Y. Bliokh, F. J. Rodríguez-Fortuño, F. Nori, and A. V. Zayats, Spin-orbit interactions of light, Nat. Photonics 9, 796 (2015).

[44] K. Yu. Bliokh and Y. P. Bliokh, Conservation of Angular Momentum, Transverse Shift, and Spin Hall Effect in Reflection and Refraction of an Electromagnetic Wave Packet, Phys. Rev. Lett. 96, 073903 (2006).

[45] M. Onoda, S. Murakami, and N. Nagaosa, Hall Effect of Light, Phys. Rev. Lett. 93, 083901 (2004).

[46] K. Y. Bliokh, Geometrodynamics of polarized light: Berry phase and spin Hall effect in a gradient-index medium, J. Opt. A 11, 094009 (2009).

[47] K. Yu. Bliokh, Geometrical Optics of Beams with Vortices: Berry Phase and Orbital Angular Momentum Hall Effect, Phys. Rev. Lett. 97, 043901 (2006). 
[48] S. Zhang and Z. Yang, Intrinsic Spin and Orbital Angular Momentum Hall Effect, Phys. Rev. Lett. 94, 066602 (2005).

[49] See Supplemental Material at http://link.aps.org/supplemental/ 10.1103/PhysRevResearch.1.032014 for the evolution of the phase, amplitude, and transverse energy flux.

[50] Data available at https://cchdo.ucsd.edu/, last accessed on 4 July 2019.

[51] M. Stojanovic and J. Preisig, Underwater acoustic communication channels: Propagation models and statistical characterization, IEEE Commun. Mag. 47, 84 (2009).

[52] M. Isakson, B. Goldsberry, and N. P. Chotiros, A threedimensional, longitudinally-invariant finite element model for acoustic propagation in shallow water waveguides, J. Acoust. Soc. Am. 136, EL206 (2014).

[53] M. B. Porter, The BELLHOP Manual and User's Guide: Preliminary Draft (Heat, Light, and Sound Research, La Jolla, CA, 2011).

[54] We used 2000 beams ranging from $-20^{\circ}$ to $20^{\circ}$ angles in the simulations.

[55] For the small dimension of our source array $(r=0.5 \mathrm{~m}$ in radius), the range difference between the individual sources, $r^{2} / L$, is smaller than $2.5 \times 10^{-4} \mathrm{~m}$ for a range $L>1 \mathrm{~km}$ or is three orders smaller than the wavelength $0.1 \mathrm{~m}$. As such, we approximate the out-of-plane sources $(y \neq 0)$ to be in plane $(y=0)$ in the simulation.

[56] J. T. Karlsen, P. Augustsson, and H. Bruus, Acoustic Force Density Acting on Inhomogeneous Fluids in Acoustic Fields, Phys. Rev. Lett. 117, 114504 (2016).

[57] R. Piazza, Thermophoresis: Moving particles with thermal gradients, Soft Matter 4, 1740 (2008).

[58] M. Škarabot, Ž. Lokar, and I. Muševič, Transport of particles by a thermally induced gradient of the order parameter in nematic liquid crystals, Phys. Rev. E 87, 062501 (2013).

[59] J. Chen, H. Cong, F.-C. Loo, Z. Kang, M. Tang, H. Zhang, S.-Y. Wu, S.-K. Kong, and H.-P. Ho, Thermal gradient induced tweezers for the manipulation of particles and cells, Sci. Rep. 6 , 35814 (2016).

[60] Z. Gong, P. L. Marston, and W. Li, Reversals of Acoustic Radiation Torque in Bessel Beams Using Theoretical and Numerical Implementations in Three Dimensions, Phys. Rev. Applied 11, 064022 (2019).

[61] Y. Long, J. Ren, and H. Chen, Intrinsic spin of elastic waves, Proc. Natl. Acad. Sci. USA 115, 9951 (2018).

[62] K. Y. Bliokh and F. Nori, Spin and orbital angular momenta of acoustic beams, Phys. Rev. B 99, 174310 (2019).
[63] A. Ciattoni, G. Cincotti, and C. Palma, Angular momentum dynamics of a paraxial beam in a uniaxial crystal, Phys. Rev. E 67, 036618 (2003).

[64] L. Marrucci, C. Manzo, and D. Paparo, Optical Spin-toOrbital Angular Momentum Conversion in Inhomogeneous Anisotropic Media, Phys. Rev. Lett. 96, 163905 (2006).

[65] G. Cincotti, A. Ciattoni, and C. Sapia, Radially and azimuthally polarized vortices in uniaxial crystals, Opt. Commun. 220, 33 (2003).

[66] N. A. Khilo, Diffraction and order conversion of Bessel beams in uniaxial crystals, Opt. Commun. 285, 503 (2012).

[67] A. Ciattoni, G. Cincotti, and C. Palma, Circularly polarized beams and vortex generation in uniaxial media, J. Opt. Soc. Am. A 20, 163 (2003).

[68] S. N. Khonina, S. V. Karpeev, S. V. Alferov, and V. A. Soifer, Generation of cylindrical vector beams of high orders using uniaxial crystals, J. Opt. 17, 065001 (2015).

[69] T. Fadeyeva, A. Rubass, Y. Egorov, A. Volyar, and G Swartzlander, Quadrefringence of optical vortices in a uniaxial crystal, J. Opt. Soc. Am. A 25, 1634 (2008).

[70] S. N. Khonina, V. D. Paranin, A. V. Ustinov, and A. P. Krasnov, Astigmatic transformation of Bessel beams in a uniaxial crystal, Opt. Appl. 46, 5 (2016).

[71] S. Hacyan and R. Jáuregui, Evolution of optical phase and polarization vortices in birefringent media, J. Opt. A 11, 085204 (2009).

[72] D. H. Zusin, R. Maksimenka, V. V. Filippov, R. V. Chulkov, M. Perdrix, O. Gobert, and A. S. Grabtchikov, Bessel beam transformation by anisotropic crystals, J. Opt. Soc. Am. A 27, 1828 (2010).

[73] D. Liu, Y. Wang, and H. Yin, Evolution properties of partially coherent four-petal Gaussian vortex beams propagating in uniaxial crystals orthogonal to the optical axis, J. Opt. Soc. Am. A 32, 1683 (2015).

[74] T. A. Fadeyeva, A. F. Rubass, and A. V. Volyar, Transverse shift of a high-order paraxial vortex-beam induced by a homogeneous anisotropic medium, Phys. Rev. A 79, 053815 (2009).

[75] A. Riaud, J.-L. Thomas, E. Charron, A. Bussonnière, O. Bou Matar, and M. Baudoin, Anisotropic Swirling Surface Acoustic Waves from Inverse Filtering for On-Chip Generation of Acoustic Vortices, Phys. Rev. Applied 4, 034004 (2015).

[76] A. Riaud, J.-L. Thomas, M. Baudoin, and O. Bou Matar, Taming the degeneration of Bessel beams at an anisotropic-isotropic interface: Toward three-dimensional control of confined vortical waves, Phys. Rev. E 92, 063201 (2015). 